\section{$\underset{\substack{\text { hommes } \\ \text { \& migrations }}}{ }$}

\section{Hommes \& migrations}

Revue française de référence sur les dynamiques

migratoires

$1278 \mid 2009$

Histoire des immigrations. Panorama régional

\title{
Entrées migratoires en Corse
}

Mise en relief de quelques spécificités

\section{Philippe Pesteil}

\section{(2) OpenEdition \\ 1 Journals}

\section{Édition électronique}

URL : https://journals.openedition.org/hommesmigrations/228

DOI : $10.4000 /$ hommesmigrations.228

ISSN : 2262-3353

Éditeur

Musée national de l'histoire de l'immigration

Édition imprimée

Date de publication : 1 mars 2009

Pagination : 62-74

ISSN : 1142-852X

\section{Référence électronique}

Philippe Pesteil, «Entrées migratoires en Corse », Hommes \& migrations [En ligne], 1278 | 2009, mis en ligne le 29 mai 2013, consulté le 28 juin 2022. URL : http://journals.openedition.org/

hommesmigrations/228; DOI : https://doi.org/10.4000/hommesmigrations.228 


\section{Entrées migratoires en Corse} Mise en relief de quelques spécificités

Par Philippe Pesteil, maître de conférences, université de Corse.

De par son histoire et sa situation géographique, la Corse a longtemps entretenu des rapports privilégiés avec son voisin italien, qui lui a apporté un fort contingent d'immigrés (immigration politique et économique), longtemps hégémonique, qui influença socialement, culturellement et politiquement l'île. Lorsque, dans les années 1960, la Corse bascule vers une nouvelle économie, de nouveaux immigrés affluent, notamment des Marocains

(surtout manœuvres et récoltants), ainsi qu'un contingent non négligeable, mais rarement mentionné, de ressortissants allemands qui investissent le secteur touristique. La question de l'immigration y reste toutefois un sujet sensible. 
En neuf millénaires de peuplement, la Corse a vu se succéder de nombreux groupes qui ont influencé l'histoire économique, sociale et culturelle de l'île. Les apports de population ne sont pas tous équivalents, qui vont des arrivées individuelles aux apports massifs et continus, et les installations ne furent pas toujours définitives, la Corse ayant été parfois une terre d'asile temporaire ou de travail saisonnier. On a pu dire qu'elle est à la fois une terre accueillante et ouverte sur l'extérieur tout en étant méfiante de ces apports étrangers. Cette double tendance à l'apparence contradictoire fonctionne de concert et permet d'appréhender les phénomènes migratoires et leurs représentations. Au recensement de 1999, l'île comptait 26000 immigrés, soit $10 \%$ de sa population, la plaçant au deuxième rang national après l'Île-de-France. Cette donne évolue puisque, en 2005, elle n'était plus qu'au $5^{\mathrm{e}}$ rang et est la seule région à connaître un recul de la proportion des immigrés. Cependant, elle est aussi la région qui comporte le plus d'immigrés dans sa population active (18,3\%), en raison notamment de l'importance des classes retraitées.

Nous avons construit nos propos à partir des grandes lignes structurantes et particularisantes de l'immigration en Corse et avons porté l'accent sur l'adéquation entre les flux migratoires et leur composition avec l'évolution des caractéristiques économiques insulaires. À signaler aussi que nous avons adopté une définition large du terme synonyme d'étrangers, la distinction entre réfugié politique et migrant pour des raisons économiques n'étant pas toujours aisée et la réalité du terrain imposant souvent de passer d'une catégorie à l'autre.

\section{Le flux italien, du quasi-monopole au tarissement des apports}

Tant par l'importance de ses effectifs et sa pérennité que pour l'influence qu'il a eue sur l'île, le flux italien mérite d'être mis en relief. Si nous n'oublions pas son impact social, nous traiterons ici d'un aspect souvent peu entrevu : les aspects politiques qui découlèrent des différents courants migratoires.

Dès le XVII siècle, l'apport d'une main-d'ceuvre toscane, principalement destinée à la construction des terrasses visant à réduire les pentes et à limiter le ruissellement, a été décisif pour la Corse, tant pour l'essor économique que pour la constitution des paysages. S'il a été gommé par la mémoire collective, il détermine la continuité des flux migratoires à but économique qui ne se démentiront pas du $\mathrm{XVII}^{\mathrm{e}}$ au milieu du $\mathrm{XX}^{\mathrm{e}}$ siècle.

L'examen de l'évolution de l'immigration italienne confirme qu'il s'agit de la présence la plus massive des étrangers sur l'île pour une période d'au moins un siècle. 
Ce n'est qu'à partir des années 1930 que la proportion des Italiens se situe sous les $90 \%$, pour remonter à $92 \%$ dans les années 1950, période de marasme économique et démographique très profond précédant l'arrivée des Marocains.

\section{Première moitié du XIX' siècle : les exilés du Risorgimento}

Les révoltes et répressions qui ponctuent la première moitié du XIX ${ }^{\mathrm{e}}$ siècle dans la péninsule italienne apportent à la Corse son lot de réfugiés, simples militants recherchés ou proscrits politiques et intellectuels. L'accueil qui leur est réservé éclaire l'expression "politiquement françaises, culturellement italiennes" qui sert à qualifier les élites locales. Une évidente proximité, souvent confortée par une même sensibilité politique, a permis aux exilés de trouver sur place un soutien moral et matériel non négligeable. C'est ainsi que la Corse abritera pour des périodes plus ou moins longues les poètes Pitro Sterdini, Flaminio Lolli, l'écrivain et militant Giovanni la Cecilia, sans compter des politiques influents parmi lesquels très temporairement Giuseppe Mazzini lui-même ${ }^{(1)}$. Parmi les plus célèbres écrivains qui laissèrent une influence importante au sein du milieu littéraire bastiais, florissant à l'époque, citons Niccolò Tommaseo et Francesco Domenico Guerrazzi, fondateur du roman historique du Risorgimento qui puisera de son épisode corse une profonde inspiration. Tommaseo, lui, grand nom de la littérature italienne du XIX siècle, va activement cuvrer, à l'instar des auteurs européens qui participent à la réhabilitation des cultures populaires rurales et y puisent un argumentaire pour prôner l'émergence des revendications nationales, à la valorisation des figures héroïques corses, notamment celle de Pascal Paoli, le "père de la patrie". Ces deux auteurs, qui insistent sur les figures historiques de l'île, célèbrent les résistances insulaires aux envahisseurs et, de façon générale, exaltent la culture rurale et son authenticité, inspirent les auteurs locaux et fournissent des arguments aux partisans du maintien des liens culturels, voire politiques avec l'Italie. Ce sentiment d'appartenance à la même culture sera repris par le mouvement autonomiste aux lendemains de la Première Guerre mondiale.

\section{Des influences politiques réciproques}

L'influence des écrivains et plus encore des hommes politiques se retrouvera dans le développement du carbonarisme qui, avec la chute de Charles $\mathrm{X}$ et l'avènement de Louis-Philippe, en qui il escomptait un allié libéral, se tourna résolument vers la cause de l'unité italienne. Avec l'échec de 1831, l'activité carbonariste en Corse vint alimenter le mouvement Giovine Italia de Mazzini. Sur le terrain, celui-ci va céder le terrain aux pinnuti, société secrète fortement semblable à la précédente. 
Les contacts et les influences entre le mouvement du Risorgimento, les carbonari italiens et les pinnuti corses sont historiquement avérées. Louis-Napoléon sera d'ailleurs élu en 1848 avec 95 \% des voix, soutenu par les pinnuti en raison de son soutien aux carbonari italiens et de sa politique d'aide à l'unité italienne. Mais l'élection d'un Corse à la tête de la France verra les perspectives d'un rapprochement politique avec l'Italie, qui accédait enfin à l'indépendance, définitivement closes.

\section{Après l'unité italienne :}

\section{une immigration essentiellement économique}

L'affirmation de ce fort courant de sympathie fini par se dissoudre avec l'unité italienne et la disparition des exilés, bientôt remplacés par des immigrés pauvres venus chercher du travail en Corse. Ainsi, les Italiens participent à tous les grands chantiers qui s'ouvrent en Corse (construction du chemin de fer, percement et élargissement des routes) ou à l'essor de l'industrie (usines de tanin, scieries...). Ils fournissent également un important contingent à l'artisanat en tant que maçons. Mais ils continuent plus classiquement à constituer une force de travail importante pour le monde rural. Soulignons leur spécialisation dans le domaine du charbon de bois où des équipes structurées autour d'un caporal réalisent l'ensemble du procès de production, de l'abattage des arbres à l'acheminement du combustible. En définitive, on les retrouve partout où il existe une offre de travail, généralement peu qualifiée, peu rémunérée et physiquement dure.

\section{De nos jours, une certaine distanciation à l'égard du voisin italien}

Quand le gouvernement italien adhère à la Triplice (1882) et les relations avec la France se tendent sous le gouvernement Crispi ${ }^{(2)}$, le regard porté sur l'Italie et ses ressortissants présents sur l'île devient tout autre. Les rapports s'apaisent après 1901 lors du rapprochement Paris-Rome et surtout durant la Première Guerre mondiale, pour se durcir à nouveau à partir de l'époque fasciste, en 1923. La Corse accueillera de nouveau de nombreux Italiens fuyant le gouvernement en place (difficile ici de discerner l'immigré proprement dit du réfugié politique). Des ressortissants italiens présents en Corse lors de l'entrée en guerre et durant l'Occupation participeront à la libération de l'île. Leur importance numérique ainsi que leur implication dans la Résistance se concrétiseront par une forte présence du parti communiste dans certaines régions : Sartène, Alta Rocca, Bastia. Cette importante présence d'immigrés italiens engendra plus d'une fois des commentaires alarmistes qui pouvaient utiliser les tensions internationales pour stigmatiser l'étranger. Le rappel incessant de la dure domination génoise fait même 
naître le terme "d'ennemi héréditaire" que l'on étend aux ressortissants italiens d'après l'unité. Le même procédé est alimenté par l'histoire récente ou l'actualité : les déboires italiens lors de la conquête de l'Éthiopie (défaite de Dogali en 1887), la très problématique réussite en Lybie, les hésitations d'alliance entre France et Allemagne, le comportement des troupes lors de la bataille de Caporetto (octobre 1917) font l'objet de commentaires ironiques et dépréciateurs dans une île à longue tradition militaire. Ces procédés visent à se distancier d'un voisin dont on a longtemps partagé l'histoire et la culture et à marquer son appartenance, désormais clairement affichée, à la nation française.

\section{Un traitement discriminant à l'égard des immigrés italiens pauvres}

En dehors des cercles érudits, l'attitude à l'encontre des immigrés pauvres varie au gré des vicissitudes de la politique internationale, de leur nombre supposé et des rumeurs véhiculées localement. La constante demeurera un sentiment global de mépris qui se concrétisera par un traitement discriminant ${ }^{(3)}$. Les surnoms, proverbes et dictons illustrent ce rapport à l'altérité qui prit longtemps les péninsulaires pour cible. Ceux-ci sont génériquement dénommés $i$ Lucchesi, le particulier valant pour le général, allusion à l'important contingent au début du flux migratoire des travailleurs originaires de la province de Lucques, en Toscane. L'interjection o Lucchisò vaut comme un rappel des origines non autochtones de l'interlocuteur ; sa variante $o$ Sardò constitue son équivalent plus usité pour la Corse-du-Sud, où la présence des Sardes domine. Raillés pour leurs pratiques gastronomiques (manghjalupini $^{\left({ }^{(}\right)}$), ils sont aussi accusés de véhiculer des maladies ${ }^{(5)}$. Le mariage avec un Italien équivalait à une déchéance, recours ultime pour celle qui, n’ayant pu trouver mieux ou s'étant montrée trop difficile, devait se contenter d'un Lucquois. À titre d'exemple, citons le proverbe encore usité : A la fine di tantu guai, un Lucchese un mancò $m a i^{(6)}$. Dans un autre registre, l'expression fà u Lucchese signifie s'abstenir durant les élections : l'engouement pour la politique communale et les enjeux locaux sont tels que le manquement à prendre parti ne peut être que l'attitude réservée à un étranger qui, par définition, ne vote pas.

\section{Une main-d'œuvre pourtant précieuse qui assure le maintien de certains secteurs d'activité}

On ne peut évoquer l'apport de l'immigration sans rappeler que la Corse est aussi une terre d'émigration : l'île connaît son sommet démographique en 1881, avec 273000 habitants, pour régresser régulièrement jusqu'à 160000 en 1960. La pauvreté en main-d'ceuvre locale a été compensée par la force de travail d'une 
péninsule dont l'économie ne parvient pas à retenir tous ses ressortissants. L'essaimage continu et touchant l'ensemble du territoire n'a pas produit de phénomène de concentration ni de ghetto. Les capacités pour le travail en montagne souvent acquises dans la région d'origine prédisposaient à l'accomplissement des tâches les plus rudes (muletiers, bûcherons, scieurs, bergers...). Cette compétence a permis de reconduire des activités qui se seraient éteintes dans les villages sans leur reprise par des familles immigrées qui y ont fait souche. Il en est de même pour les activités maritimes dont on sait le peu d'engouement qu'elles rencontraient en Corse, à l'exception du Cap : la pêche est souvent pratiquée par des familles d'origine napolitaine ou sarde. En ville, elles fournissent nombre de petits commerçants, artisans en particulier dans les métiers du bâtiment et des finitions. L'anecdote de l'Italien débarqué avec pour tout bien une truelle et qui possède désormais une entreprise BTP ayant pignon est devenue une réalité.

\section{Un long cheminement vers l'intégration sociale}

De nombreuses familles corses ont désormais lié des alliances avec au moins un descendant d'immigré italien ${ }^{(7)}$. Des liens familiaux ont été fréquemment maintenus avec la parentèle restée en Toscane ou en Sardaigne qui s'intègrent dans une nouvelle façon de vivre ses origines étrangères. Des voyages réguliers permettent aux enfants d'entretenir une familiarité avec la culture des ascendants et d'appré- 
hender des modes de fonctionnements sociaux différents. Des échanges de tous ordres (alimentaires, informationnels, matériels...) perpétuent une spécificité qui individualise l'identité dans le panorama local. Enfin la carte du culturel, parfois porté par les associations et les manifestations qu'elles réalisent (Semaine du cinéma italien à Bastia, jumelages...), permet d'établir un lien entre une communauté, son héritage culturel populaire ou savant et la société corse.

Cette donne ne met pas à l'abri des rappels à destination vexatoire. Une expression de la région de Sartène ne dit-elle pas sò di a carrittata ${ }^{(8)}$ pour désigner des familles d'origine italienne? Le patronyme suffit pour les initiés à signaler l'ascendance toscane ou sarde. Personne n'est à l'abri de ce phénomène qui peut s'abattre aussi bien sur ceux ayant gravi l'échelle sociale que sur ceux issus d'une déjà lointaine immigration. Il s'agit d'une tendance très répandue qui peut surgir à la moindre tension dans un échange ou comme argumentaire ad hoc pour discréditer la légitimité d'une prise de parole. On est ici dans le parfait exemple d'un état provisoire caractérisant une génération, éternisé en stigmate transmissible. Le phénomène tend à s'atténuer en raison d'un partiel renversement des stigmates qui favorise la diversité des origines et valorise les identités composites, surtout quand elles sont latines. En outre, la question italienne est passée d'actualité ; l'immigration régresse et n'est plus alimentée par un flux continu, et la population n'ayant pas acquis la nationalité française est la plus âgée parmi l'ensemble des ressortissants étrangers (58 ans, pour 42 ans en moyenne). L'attention s'est reportée sur la communauté marocaine.

\section{Des flux diversifiés caractéristiques de l'économie modeme}

C'est dans les années 1960 que la Corse bascule vers une économie fondée sur l'agriculture littorale et le tourisme, tout en confortant une prépondérance marquée du secteur tertiaire. Il s'agit d'un bouleversement profond qui voit l'accélération de l'urbanisation et le dépeuplement de l'intérieur. La viticulture et les agrumes exploités en plaine ainsi que la politique d'équipements destinée à rattraper les retards de développement vont justifier le recours à une main-d'ceuvre immigrée.

\section{L'anivée massive des pieds-noirs à la suite de l'indépendance algérienne}

Si certains Français du Maroc firent l'acquisition de terres en Corse dès 1957, ce fut l'indépendance de l'Algérie et l'exode des pieds-noirs qui bouleversa la donne. En 1966, on dénombrait environ 15000 rapatriés arrivés dans l'île, dont 4500 
pour la seule année 1962. Plus de la moitié étaient originaires d'Algérie et une partie avait une ascendance insulaire. Près de 40 \% d'entre eux s'installèrent en ville (Ajaccio, Bastia, Bonifacio, Porto-Vecchio...), investissant le secteur commercial (hôtellerie, garages automobiles, entreprises du bâtiment...), y apportant un dynamisme certain et de nouvelles pratiques commerciales, en particulier le recours au crédit. La plupart, néanmoins, choisirent l'agriculture et plus particulièrement la viticulture dans la plaine orientale. Leur maîtrise des techniques modernes révolutionna une agriculture insulaire en état de déliquescence avancée. Ce sont eux qui feront appel à la main-d'ceuvre nord-africaine. Le secteur Aléria/Ghisonaccia voit alors se concentrer les efforts de développement ainsi que les nouvelles populations immigrées venues travailler en qualité d'ouvriers agricoles.

\section{Une dominante maghrébine marocaine}

La forte présence des Marocains en Corse est un des facteurs les plus caractéristiques de l'immigration insulaire dans l'ensemble national. Avec environ 11000 ressortissants, ils représentent $42 \%$ des étrangers (12\% au niveau national) ${ }^{(9)}$. Il s'agit d'une communauté à fort taux de rotation car si les effectifs restent stables les arrivées n'ont pas baissé. Il s'agissait au départ de répondre à des besoins saisonniers correspondant aux récoltes viticoles et agrumicoles (clémentines); l'offre de travail s'est à présent stabilisée et s'étale sur toute l'année, malgré les difficultés à obtenir un permis de séjour et à pérenniser un emploi qui tend à se raréfier. Le taux de chômage des Marocains est élevé et se situe à un quart des actifs. L'absence fréquente de diplôme entrave la recherche d'un emploi, phénomène patent en ce qui concerne les femmes. Seulement 15 \% des Marocaines entre 20 et 59 ans ont un emploi, souvent peu qualifié (employée de maison, personnel de nettoyage, assistante maternelle). Il s'agit d'une population jeune : 36 \% des natifs marocains ont moins de 30 ans, ce qui explique le faible taux d'acquisition de la nationalité française $-7 \%$, contre $25 \%$ pour l'ensemble national. Essentiellement employée dans l'agriculture et le bâtiment, cette population se concentre sur les bassins d'emploi concernés par ces activités : Aléria/Ghisonaccia, Porto-Vecchio, Bastia, Ajaccio, Calvi. Si les Marocains sont nombreux dans la capitale de la Haute-Corse, ils sont en moindre proportion en Corse-du-Sud, où les Tunisiens et les Italiens sont mieux représentés.

\section{La difficile intégration de l'immigration marocaine}

Outre la forte densité marocaine sur certains quartiers (Citadelle de Bastia) ou portions du territoire, le mode de résidence traduit une certaine promiscuité. Entre 20 et 40 ans, 1 immigré sur 6 vit en communauté pour une proportion régionale de 1 sur 25. Ce comportement est adopté souvent par les hommes seuls, soit célibataires 
soit ayant laissé la famille au Maroc ; c'est le cas de 20 \% des hommes de plus de 15 ans. Les femmes, quant à elles, sont en couple à $80 \%$ (57 \% seulement pour les hommes), souvent venues rejoindre leur conjoint déjà sur place. Elles sont mariées à $93 \%$ avec un ressortissant de la même nationalité ; seules les Tunisiennes présentent un taux d'endogamie supérieur, avec $97 \%$. En outre, dans un autre registre, des travaux en linguistique ont explicité l'usage de l'arabe comme élément diffé-

\section{Régulièrement, des attentats non revendiqués sont commis à l'encontre de véhicules, commerces, appartements, mosquées ; le consulat du Maroc a plusieurs fois été visé, ainsi que l'association antiraciste Ava Basta.} renciateur, affirmation de la séparation et de la différence. La distinction est très nette chez les Marocains entre la langue française, assimilée à la réussite et la modernité et possédant une meilleure valeur fonctionnelle, et la langue corse, tenue comme vernaculaire, traditionnelle et rurale. Cette tendance en matière d'utilisation de sa langue d'origine et d'acquisition de la locale ne se retrouve pas par exemple chez les Portugais, peu portés à construire leurs frontières de groupe sur la clôture étanche des autres identités ${ }^{(10)}$. L'importance numérique, renforcée par cette tendance au regroupement, est stigmatisée par une partie de la population corse qui y voit un risque d'invasion et, pour quelques-uns, justifie des actes d'intimidation.

\section{Les Maghrébins, une population stigmatisée, victime d'actes racistes}

En 2001 et 2002, des incidents éclatent entre jeunes, et des actions ponctuelles sont perpétrées à l'encontre des ressortissants marocains. Un groupuscule clandestin se constitue et commet des plasticages signés : "I Clandestini Corsi" ; il sera rapidement démantelé et ses membres, jugés. D’autres groupes (OSC, Corsica Cristiana) se sont également signalés par des actes de propagande (distribution de tracts). Régulièrement, des attentats non revendiqués sont commis à l'encontre de véhicules, commerces, appartements, mosquées ; le consulat du Maroc a plusieurs fois été visé, ainsi que l'association antiraciste Ava Basta. Les Marocains, et les Maghrébins de façon générale, sont accusés de trafic de drogue et plus globalement de perpétrer toute sorte de délits et actes d'incivilité. Les murs de Corse sont régulièrement bombés ou tagués d'inscriptions xénophobes ciblant cette communautét ${ }^{(11)}: \operatorname{IAF}^{(12)}$ est le slogan le plus courant qui apparaitt dès 1976 et se répand à partir de 1982. Si de réelles tensions existent, des entretiens réalisés auprès de ressortissants marocains à Ghisonaccia révèlent aussi une certaine satisfaction d'être et de vivre en Corse ${ }^{(13)}$. 
Même s'il faut tenir compte d'une évidente réserve à se confier, il apparaît que l'île est appréciée pour son climat, sa nature, sa tranquillité( ${ }^{(14)}$ par rapport au continent, perçu comme un territoire d'insécurité. De façon générale, les Corses sont décrits comme accueillants, la vie sur place est agréable. Parmi les inconvénients, la difficulté à se loger, la cherté des prix sont fréquemment cités. Les conditions de travail sont peu évoquées. Les jeunes générations, cependant, plus sensibles au contexte ou plus concernées, avancent le manque d'infrastructures ludiques, les tensions entre communautés, le chômage. Cette dernière difficulté s'atténue en partie avec l'obtention de la nationalité française.

\section{Une présence originale : les Allemands de Corse}

Cependant, l'immigration en Corse ne peut se réduire à des nationalités venues fuir la misère et s'employer dans le bâtiment et le salariat agricole. Le cadre de vie a aussi attiré une population européenne en mal de nature préservée et à la recherche d'un rythme de vie moins soutenu que dans les territoires industrialisés.

En matière d'immigration, on pense rarement à évoquer les ressortissants allemands, désormais troisième nationalité européenne in situ et dont les effectifs (2,5\%) demeurent stables, venus s'installer sur l'île à la recherche d'une qualité de vie autre. On a peu de données précises sur cette communauté qui se caractérise par sa discrétion, mais que l'on retrouve très active dans des domaines liés à la nouvelle économie.

Très peu nombreux dans les années 1950, les Allemands arrivent individuellement ou en petits groupes, attirés par une destination méditerranéenne où ils découvrent une nature aux multiples aspects : mer, montagne, plage, forêts, climat tempéré... Leur venue coïncide, à partir des années 1970, avec ce qu'on a appelé la "vague hippie" et correspond localement à l'ouverture vers une économie du tourisme. La mise en service d'une ligne régulière Gênes-Bastia à partir de 1968 va contribuer à attirer vers la Corse de nombreux touristes allemands, dont certains, séduits par la beauté du pays et les perspectives de réaliser un rêve bucolique, demeureront sur place. Ils s'éparpillent alors sur le territoire, et en particulier le long de l'axe Bastia/Porto-Vecchio sur la plaine orientale. Ils recherchent souvent les habitats isolés entre village et littoral pour mettre en pratique des principes de vie proches de la nature. Leur concentration est particulière sur les communes allant de Folelli à Ghisonaccia, en passant par Linguizetta. Ceux tentés par l'expérience villageoise embrasseront la vie de berger, avec des fortunes diverses. Selon les individus, le rêve arcadien pourra lentement se concrétiser dans un austère dénuement doublé d'isolement. Les puristes perpétuent volontairement une existence faite de petits travaux et de succession de récoltes (clémentines, châtaignes). D'autres, bénéficiant d'une réussite devenue référentielle en matière agricole, exportent leur production à l'international. 


\section{Tourisme : secteur d'activité privilégié des Allemands}

Beaucoup s'investissent également dans le tourisme comme activité d'appoint en travaillant comme salariés dans les structures de vacances. On les trouve ainsi employés saisonniers dans les six camps de naturistes qui s'égrainent de Moriani à Porto-Vecchio. Leur compétence linguistique, peu partagée en Corse, y compris en anglais, leur assure des embauches qui répondent aux besoins de la clientèle. Certains mettent aussi en place des formules $B$ and $B$, type d'hébergement convivial chez l'habitant qui a tardé à se mettre en place sur l'île.

\section{Les Allemands, précurseurs du tourisme naturiste en Corse}

Devenue une destination touristique prisée, la Corse va inciter des investisseurs d'outre-Rhin à répondre à une demande particulière en matière de structures d'accueil. Ainsi, les initiateurs du nudisme se rendront acquéreurs de vastes surfaces pour y installer leur structure, ce qui n'enthousiasma pas la totalité des habitants des communes concernées. Dans un premier temps, ce tourisme sera considéré comme économiquement sans impact sur la société locale, voire moralement dangereux, et sera l'objet de nombreuses critiques. Par la suite, un modus vivendi semble avoir été trouvé et l'offre s'est intégrée dans le panel constitutif du tourisme insulaire. Avec le recul, on réalise même que les espaces devenus camps de naturistes ont été ceux qui ont le mieux résisté au développement de la construction accélérée du littoral et au bouleversement des écosystèmes. On retrouve ce souci de la préservation naturelle dans les activités de production, et la volonté de bâtir une économie agricole non intensive et basée sur le respect de l'environnement caractérise la démarche entreprise. Cette sensibilité écologique de la première heure a accéléré une prise de conscience sur la fragilité de l'environnement et l'intérêt de sa préservation.

\section{L'immigration allemande aujourd'hui}

On assiste à présent à l'arrivée d'un nouveau public plus fortuné, moins porté au renoncement. Désireux d'acquérir une résidence secondaire pour une retraite ensoleillée ou pour disposer d'un habitat de vacances qui soit aussi un investissement, ces nouveaux arrivants allemands couvrent l'ensemble du territoire sans exclusive. Soucieux de tranquillité et de nature, ils n'hésitent pas à investir dans une maison de village, contribuant à maintenir les maigres effectifs du rural. Majoritairement plus âgés que leurs prédécesseurs des années 1970, ils ne rompent pas les liens avec leur pays d'origine avec lequel ils partagent leur temps.

Malgré des itinéraires non concertés, des habitats disséminés, l'absence apparente de désir de s'instaurer en communauté, les ressortissants allemands ont constitué 
un réseau d'interconnaissance et d'entraide discret mais efficace. Les liens conservés avec l'Allemagne sont un soutien logistique parfois précieux ; le bouche-àoreille permet d'attirer de nouveaux venus désireux de découvrir une île si prisée par les touristes de ce pays.

La population corse est longtemps restée réservée à l'égard de cette présence insolite et pittoresque. Entre indifférence, amusement et agacement, les attitudes varient en fonction des individus et des rapports entretenus avec ces ressortissants. Leur réputation bohème, l'inattention à des paramètres esthétiques et différenciateurs locaux (vêtements, voitures), des pratiques marginales supposées ou affichées (consommation de haschich), les identifient parmi les autres communautés.

\section{L'immigration en Corse de nos jours : un sujet toujours sensible}

La question de l'immigration demeure un sujet sensible dans une île où une partie des habitants considère que l'État instrumentalise les mouvements de population pour noyer la population endogène sous la masse des nouveaux arrivants. La situation démographique de la Corse ne vient pas calmer ces craintes qui se développent dans un contexte de crise identitaire, de marasme économique et de précarité sociale. La quête identitaire et mémorielle, le souci de reconnaissance politique et/ou culturelle d'une partie de la population corse peuvent être interprétés à travers un sentiment de concurrente dû à la présence d'autres minorités sur le territoire. On ne peut pourtant occulter un passé d'accueil et d'aide aux plus démunis qui ont accosté sur ces rives en quête d'un travail ou fuyant les conflits. Serbes et juifs de Syrie pendant la Première Guerre mondiale, Russes blancs, républicains espagnols figurent parmi les groupes qui ont trouvé un refuge provisoire ou définitif sur l'île, venant rejoindre Italiens, puis Maghrébins et Portugais aspirant à de meilleures conditions de vie. De nombreuses familles ont ainsi pu réaliser sur place une ascension sociale qui leur paraissait irréalisable chez elles. La somme de travail investie par les vagues d'immigrés dans les réalisations et les productions de toutes sortes a contribué également à relativiser l'impact de l'émigration et de l'effondrement de l'agro-pastoralisme sur la société. Il apparaît très clairement que les apports successifs dépendent de la position géostratégique de la Corse dans l'ensemble euro-méditerranéen et qu'elles contribuent à la mise en place et à l'adaptation locale d'une économie mondialisée. La fragilité du tissu local très soumis aux variations saisonnières et conjoncturelles (tourisme, agriculture, construction) agit aussi sur les conditions de vie des étrangers et les possibilités de 
choisir des stratégies d'intégration. D'autres nationalités arrivent à présent qui reflètent les évolutions des flux migratoires européens. Les natifs d'Europe de l'Est, les Asiatiques, les ressortissants de l'Afrique subsaharienne, encore peu nombreux récemment, se signalent dans les zones urbaines. Il est difficile de dire si la société corse sera en état de les accueillir et quelle sera la qualité de la réception. Pourrontils comme leurs prédécesseurs italiens et malgré les attitudes parfois hostiles s'adapter à une société au fonctionnement complexe?

\section{Notes}

1. Ettori, Fernand, "Entre la France et l'Italie", in Le Mémorial des Corses, t. 3, Albiana, Ajaccio, 1982, p. 134-165.

2. Guichonnet, Paul, L'Italie. La monarchie libérale. 1870-1922, Hatier, Paris, 1969.

3. Rey, Didier, "Italie. La question italienne en Corse (de 1882 à 1915)", in Dictionnaire historique de la Corse, Albiana, Ajaccio, 2006, p. 522.

4. Mangeurs de lupins.

5. Rovere, Ange, "Italophobie", in Le Mémorial des Corses, t. 3, op. cit., p. 340-344.

6. "À la fin de tant de malheurs, un Lucquois ne manqua jamais."

7. Il est à noter que de nombreux citoyens français bénéficient de la double nationalité et continuent par exemple à voter en Italie.

8. Littéralement : "ils sont de la charretée". Allusion aux temps où les travailleurs italiens employés à la journée étaient chargés sur un véhicule à cheval par le patron qui les emmenait sur le lieu de travail. La région de Sartène représente un territoire où les clivages ont longtemps été parmi les plus accusés de Corse. Avoir un ancêtre qui a été vu sur la charrette continue de suivre les familles d'origine étrangère.

9. Les données statistiques qui suivent sont extraites de l'Atlas des populations immigrées en Corse, Insee/Fasild, 2005.

10. Géa, J.-M., "Pratiques, représentations et intégration linguistiques chez les migrants en Corse", in Histoire et mémoire des immigrations en région Corse, Acsé, avril 2008, p. 45-56.

11. Bertoncini, Pierre, "Graffiti bombé et territoire corse (1973-2003)", thèse en anthropologie soutenue à l'université de Corse, (sous la dir. de Philippe Pesteil), juin 2005.

12. I arabi fora: les arabes dehors.

13. Cf. Pesteil, Philippe, Histoire et mémoire des immigrations en région Corse, op. cit., p. 26-33.

14. Le terme de "tranquillité" revient constamment chez les enquêtés pour qualifier le contexte local. 